\title{
Vegetation Patterns Along a Rainfall Gradient
}

\author{
Ehud Meron ${ }^{\mathrm{a}, \mathrm{b}}$ Erez Gilad ${ }^{\mathrm{b}, \mathrm{a}}$ Jost von Hardenberg ${ }^{\mathrm{c}}$ \\ Moshe Shachak ${ }^{\mathrm{d}}$ Yair Zarmi ${ }^{\mathrm{a}, \mathrm{b}}$ \\ a Department of Solar Energy and Environmental Physics, BIDR, Ben-Gurion \\ University, Sede Boker Campus 84990, Israel \\ b Department of Physics, Ben-Gurion University, Beer Sheva 84105, Israel \\ ${ }^{\mathrm{c}}$ CIMA, University of Genoa, via Cadorna 7, I-17100 Savona, Italy \\ d Mitrani Department of Desert Ecology, BIDR, Ben-Gurion University, Sede \\ Boker Campus 84990, Israel
}

\begin{abstract}
A continuum model for vegetation patterns in water limited systems is presented. The model involves two variables, the vegetation biomass density and the soil water density, and takes into account positive feedback relations between the two. The model predicts transitions from bare soil at low precipitation to homogeneous vegetation at high precipitation through intermediate states of spot, stripe and gap patterns. It also predicts the appearance of ring-like shapes as transient forms toward asymptotic stripes. All these patterns have been identified in observations made on two types of perennial grasses in the Northern Negev. Another prediction of the model is the existence of wide precipitation ranges where different stable states coexist, e.g. a bare soil state and a spot pattern, a spot pattern and a stripe pattern, and so on. This result suggests the interpretation of desertification followed
\end{abstract}


by recovery as an hysteresis loop and sheds light on the irreversibility of desertification.

Key words: Vegetation patterns, mathematical modelling, positive feedback, state coexistence, hysteresis, desertification.

\section{Introduction}

Geophysical patterns range over a wide range of scales, from the size of a small heaps of desert sand to the size of large scale jets and eddies in the atmosphere. Sometimes scale invariance over a few orders of magnitude is observed, such as in river networks, and coherent structures in atmospheric and oceanic flows. More often a characteristic length scale is preferred as in the case of convective cells [1], river bed ripples [2], aeolian sand ripples [3], stone stripes [4], and banded vegetation [5].

Vegetation bands tend to develop on gentle hill-slopes in arid and semi-arid regions. They have been observed in Africa, Australia, Asia and South America [5]. The bands may consist of trees, shrubs and perennial grass, or combinations thereof. The characteristic length scale (width of band and inter-band spacing) is of the order of 10 to $10^{2} \mathrm{~m}$. The bands are most often oriented perpendicular to the hill slope but the orientation may be affected by a dominant wind direction. The phenomenon has attracted considerable interest in the

Email addresses: ehud@bgumail.bgu.ac.il (Ehud Meron), gilade@bgumail.bgu.ac.il (Erez Gilad), jost@cima.unige.it (Jost von Hardenberg), shachak@bgumail.bgu.ac.il (Moshe Shachak), zarmi@bgumail.bgu.ac.il (Yair Zarmi). 
past few years and motivated both field studies [6] and mathematical modelling [7-14]. The models reproduced vegetation bands as well as isotropic patterns in plain areas such as spots and labyrinths. The studies of these models suggest that vegetation pattern formation is a symmetry breaking phenomenon, occurring even if the system and the forces it is subjected to are completely uniform.

The symmetry breaking of vegetation patterns, manifested by the appearance of a particular length scale, has been attributed to a finite wavenumber instability of a homogeneous state (bare soil or uniform vegetation) $[15,1]$. The mechanism of this instability is a positive feedback between biomass (amount of plant matter) and soil water [16]. The more soil water the faster the plant growth, and the larger the plant the more soil water available to it. Two main processes are responsible for this positive feedback. The first one is the drainage of surface water into vegetated areas, where physical and biological soil crusts are absent and the infiltration of surface water into the soil is higher [17]. The second feedback process is water uptake by the plant's roots which is higher with larger plants having longer roots.

In this paper we present a model that was briefly introduced in Ref. [18]. We describe the states along a rainfall gradient that the model predicts and show the existence of rainfall ranges where two or even three stable states coexist. The paper supplements Ref. [18] with additional analyses, simulations, observations and discussions.

We proceed in Section 2 to the presentation of the model equations. In Section 3 we study uniform solutions of the equations and their stability. In Section 4 we present numerical simulations of the model, and in Section 5 we compare 
simulations with field observations. We conclude with a discussion in Section 6.

\section{The model equations}

The system we consider is described by two dynamical variables, the plant biomass density, $N(\mathbf{x}, t)$, and the water density, $W(\mathbf{x}, t)$, in units of $K g / m^{2}$. The biomass variable represents the amount of plant matter while the water variable represents the total water density in the top soil layer, including the water taken up by the roots. The evolution in time of these variables is described by the following model equations:

$$
\begin{aligned}
\frac{\partial N}{\partial t} & =\frac{G W}{1+S W} N-C N^{2}-M N+D_{n} \nabla^{2} N \\
\frac{\partial W}{\partial t} & =P-I(1-R N) W-F W^{2} N+D_{w} \nabla^{2}(W-B N) .
\end{aligned}
$$

The term $\frac{G W}{1+S W} N$ in Eq. (1) describes plant growth at a constant rate, $G / S$, for saturated soil, and at a rate that grows linearly with $W$ for dry soil. The term $-M N$ accounts for mortality and grazing, and the quadratic term $-C N^{2}$ represents saturation due to limited nutrients. The spread of plants, both by clonal reproduction and by seed dispersal is modelled by the diffusion term $D_{n} \nabla^{2} N[15]$

Eq. (2) contains a source term, $P$, representing precipitation, and a loss term $-I(1-R N) W$. In the absence of plants, the loss rate of water due to factors such as evaporation and drainage can be modelled by a term $-I W$. Vegetation coverage reduces this loss through an increase in local water infiltration and reduced evaporation due to shading. Local uptake of water by plants (mostly 
transpiration) is modelled by the term $-F W^{2} N$ (this form was motivated by transpiration curves appearing in Ref. [19]). The transport of water in the soil is modelled by Darcy's law which states that the water flux $\mathbf{J}$ is proportional to the gradient of the water matric potential $\phi, \mathbf{J} \propto-\nabla \phi,[19]$. To account for the water uptake by the roots we assume the form $\phi=\phi_{0}-B N$, where $\phi_{0}$ is the matric potential for bare soil. The root system is regarded here as a transport mean for the plant to collect water from its surrounding. In the numerical simulations we used the simple form $\phi_{0}=W$ (assuming constant hydraulic diffusivity. See Ref. [19]). The temporal change of $W$ due to transport, $-\nabla \cdot \mathbf{J} \propto$ $\nabla^{2} \phi$, gives the Laplacian term in Eq. (2). The water dependent, plant-growth term in Eq. (1) and the terms containing the parameters $R$ and $B$ in Eq. (2) describe positive feedback effects of water and biomass.

Equations (1) and (2) can be brought to the non-dimensional forms

$$
\begin{aligned}
\frac{\partial n}{\partial t} & =\frac{\gamma w}{1+\sigma w} n-n^{2}-\mu n+\nabla^{2} n \\
\frac{\partial w}{\partial t} & =p-(1-\rho n) w-w^{2} n+\delta \nabla^{2}(w-\beta n)
\end{aligned}
$$

by introducing non-dimensional variables and parameters as follows: $n=$ $(C / I) N, w=(F / C) W, \tilde{t}=I t, \tilde{\mathbf{x}}=\left(I / \sqrt{D_{N}}\right) \mathbf{x}, \gamma=(C / I F) G, \sigma=(C / F) S$, $\mu=I^{-1} M, p=(F / I C) P, \rho=(I / C) R, \delta=D_{W} / D_{N}, \beta=\left(I F / C^{2}\right) B$. The tilde signs of the new time and space variables were dropped from Eqs. (3) and (4).

Realistic parameter values can be determined following Refs. [19,20]. In the simulations we have chosen $G=0.04 \mathrm{~mm}^{-1} \mathrm{yr}^{-1}, S=0.01 \mathrm{~mm}^{-1}, C=4$ $\mathrm{m}^{2} /(\mathrm{kg} \mathrm{yr}), M=0.8 \mathrm{yr}^{-1}, I=4 \mathrm{yr}^{-1}, R=1.5 \mathrm{~mm}^{2} \mathrm{~kg}^{-1}, F=0.025 \mathrm{~m}^{4} /\left(\mathrm{kg}^{2}\right.$ yr), $D_{n}=5 \cdot 10^{-4} \mathrm{~m}^{2} \mathrm{yr}^{-1}, D_{w}=5 \cdot 10^{-2} \mathrm{~m}^{2} \mathrm{yr}^{-1}$, and $\beta=I F B / C^{2}=3$. For 
the parameter $p$ a range of values will be considered.

\section{Uniform solutions and their stability}

The model equations (3) and (4) have two uniform stationary solutions: (i) $\mathcal{B}: n=0, w=p$ describing a bare soil, and $\mathcal{V}: n=n_{0}, w=w_{0}$ describing a uniform vegetation state (the values of $n_{0}$ and $w_{0}$ will be evaluated numerically, the explicit analytical forms are too complicated to display). It is first instructive to consider the stability of these states to uniform perturbations. At low precipitation $p$ the bare soil state is stable, but as $p$ is increased it loses stability to the uniform vegetation state. The nature of the instability depends on the parameter $\rho$ as shown in Fig. 1. This parameter controls the positive feedback effect between biomass and water associated with water loss reduction; the higher the biomass the smaller the loss. For $\rho<\rho_{c}$, where $\rho_{c}$ is evaluated numerically, the positive feedback effect is small and the bare soil state loses stability to uniform vegetation in a supercritical bifurcation at $p=p_{c}=\mu /(\gamma-\mu \sigma)$. For $\rho>\rho_{c}$ the instability becomes subcritical; a range of $p$ exists where both the bare soil and the uniform vegetation states are stable. The coexistence of the two states results from the big reduction in the water loss as the vegetation grows [21].

We now consider the stability of the two uniform states to nonuniform perturbations. The bare state is found to be stable to nonuniform perturbations and the first instability it encounters is the uniform (or zero wavenumber) instability discussed above. Fig. 2 shows growth rate curves below the instability $\left(p<p_{c}\right)$ at the instability $\left(p=p_{c}\right)$ and above it $\left(p>p_{c}\right)$. The uniform vegetation state, however, may undergo a finite wavenumber instability as $p$ 
increases from low values beyond $p_{1}$ or decreases from high values below $p_{2}$. Fig. 3 shows growth rate curves for the instability at $p=p_{2}$. The physical picture of this instability is the positive feedback due to water uptake by the plant roots. A plant that has slightly longer roots then a nearby plant take up more water from the soil and leave less water for its neighbor. As a result it grows faster, takes up even more water and inhibits the growth of the neighbor plants.

\section{Nonuniform solutions of the model and their significance}

The two uniform states, $\mathcal{B}$ and $\mathcal{V}$ are stable at low and high $p$ (precipitation) values, respectively. At intermediate $p$ values stable nonuniform solutions of Eqs. (3) and (4) are numerically found. At relatively low $p$ values, nonuniform solutions representing spot patterns appear. At higher $p$ values stable solutions describing stripes or labyrinths appear. At yet higher $p$ values solutions representing gaps in a uniform coverage are found. The spot, stripe and gap patterns reflect optimal self-organization of the system with respect to water resources. Spot patterns are the preferred patterns at low $p$ since water uptake from adjacent bare areas can be made in all directions. Stripes, which have only two directions to extract water from, need higher precipitation values. Spot, stripe and gap patterns have also been found in Refs. [12-14].

Fig. 4 summarizes all the stable states of the model along the rainfall gradient,

$0<P<480 m m(0<p<0.6)$, by displaying the spatial average $\langle n\rangle$, of the biomass density as a function of $p$. The figure was created by scanning $p$ upward and downward starting the simulation at each value of $p$ with the pattern produced at the previous $p$ value. In this way ranges of $p$ where two or 
even three stable solutions were identified. Increasing $p$ from very low values we found the bare soil state to remain stable all the way till $p=p_{c}$. But starting with a spot pattern at $p>p_{c}$ and decreasing $p$, the transition to the bare soil state occurred at $p_{0}<p_{c}$, implying a coexistence range, $p_{0}<p<p_{c}$, where both bare soil and spots are stable states. Similarly, a coexistence range of uniform vegetation and gap patterns was found at high $p$ values. Pattern states may not only coexist with uniform states but also with other pattern states. A spot pattern may coexist with a labyrinthine pattern and the latter with a gap pattern. A small $p$ range was even found where all three patterns coexist. Figs. 5 shows coexistence of spots and labyrinths at the same precipitation value. In Fig. 5(a) initial conditions were chosen such that one half of the system converges to a spot pattern and the other half to a stripe pattern. In Fig. 5 (b) the intial condition is the unstable uniform vegetation state with random perturbations imposed on it. In this case the system converges to a mixture of spots and stripes. We also found a range of $p$ values were all the three patterns coexist as stable states. In the field this coexistence of stable states can be attributed to the positive feedback effect associated with the roots. As an example, under relatively dry conditions a small plant may not survive because of its short roots and the incapability of the plant to collect water from its surrounding. However, under the same environmental conditions, a big plant with a well developed root system may survive.

The three pattern states, spots, labyrinths and gaps, describe asymptotic patterns, that is, patterns the system converges to at long times. Different initial states may lead to the same type of asymptotic pattern but the transient behaviors will obviously be different. Figs. 6 show the development of a spot pattern from (a) an initial uniform vegetation state and (b) an initial state con- 
sisting of a few spots. Fig. 7 shows the development of a stripe (labyrinthine) pattern, at higher precipitation value, from similar initial conditions. The developments of spot and stripe patterns from isolated initial spots involve transient rings. The appearance of rings can be interpreted as follows: as an initial spot expands beyond a characteristic size the plants at the center of the spot can no longer take up water (using their roots) from the patch surrounding and die out, thus forming a ring.

The coexistence of stable vegetation states sheds new light on desertification phenomena [18]. Desertification is a long lasting or irreversible decrease in biological productivity caused by climatic changes (such as drought) or by human activities (such as over-grazing or clear-cutting) [22]. In terms of the model, desertification caused by a prolonged drought can be understood as follows. Consider the precipitation range $p_{0}<p<p_{c}$ where a bare soil coexists with a spot pattern. A soil covered with a spot pattern in this range of precipitation will dry out when subjected to a prolonged drought that reduces $p$ below $p_{0}$. The resumption of rainfall to the original precipitation level, however, will not be followed by a recovery of the vegetation because the coexisting bare soil continues to be stable. A particulary rainy period is needed for the vegetation to spontaneously recover (and complete the hysteresis loop). Desertification due to human activities, such as grazing, can also be related to coexistence of stable states. From the point of view of dynamical systems theory, an unstable solution should exist between the two stable ones. This solution or state introduces a threshold for the flow in phase space. Sub-threshold perturbations (e.g. grazing) of a spot pattern in the range $p_{0}<p<p_{c}$ will decay out and the spot pattern will recover. However, super-threshold perturbations (i.e. over-grazing) will grow and drive the system to the coexisting stable bare-soil 
state.

Coexistence of states not only implies vulnerability to desertification, but also the possible recovery of desertified regions with appropriate human intervention. It is therefore significant to know whether a given area represents a system with a single stable state or with coexistence of two or more stable states. To highlight this geographical property we suggested in Ref. [18] a new classification of aridity based on the inherent vegetation states of the system. According to this classification a hyper-arid region is characterized by a single stable state, the bare soil state. An arid region is characterized by coexistence of a stable bare soil and a stable pattern state (spots), and so on (see Fig. 4). Thus, a region with patches of vegetation which is classified as arid, is vulnerable to desertification, and a bare-soil region, also classified as arid, is recoverable. A bare-soil region classified as hyper-arid is not recoverable and attempts to recover vegetation will fail.

\section{Model simulations vs. field observations}

Most observations of vegetation patterns focused on vegetation bands that develop on gentle slopes. Such patterns are reproduced by models that include the effect of runoff water $[7,18,14]$. Here we present observations of two perennial grasses, Paspalum vaginatum and Poa bulbosa, made in flat areas in the Northern Negev where average annual rainfall is about $200 \mathrm{~mm}$.

Fig. 8a shows a labyrinth-like pattern of Paspalum vaginatum. Closeups at different locations of the same area are shown in Figs. 8b,c,d. They reveal the three pattern states, spots, stripes and gaps that the model predict (compare 
with the insets in Fig. 4). In this case all three patterns where observed in the same area on the same day. Figs. 9a,b show scattered spots and ringlike shapes of Poa bulbosa. Scattered spots and rings appear in the model simulations as transients as Figs. 6 show. The simulations were carried out with constant values of the precipitation parameter $p$, while in nature the rainfall is time dependent. We expect dry periods that follow rainy ones to extend the durations of these transients.

\section{Conclusion}

The model presented in this paper takes into account the positive feedback between biomass and water due to water up-take by the plants' roots, but does not include the positive feedback associated with water drainage into vegetation patches. The latter feedback effect has been included in a recent study by Rietkerk et al. [14] who split the water variable into two independent variables; soil water and surface water. This model does not take into account the roots effects but produces the same sequence of states; bare soil, spots, stripes, gaps and uniform vegetation. This sequence has also been found in a single-variable model (biomass only) that takes into account resource competition effects [11-13].

The conclusion we may draw from these studies is that the sequence of states along the rainfall gradient, bare soil, spots, stripes, gaps and uniform vegetation, is likely to be a robust feature of planar water limited systems and that the mechanism behind it is a positive feedback between biomass and water. The particular positive feedback mechanism (water up-take by roots or water

drainage) appears to be of secondary importance as far as the sequence of 
states is concerned.

In general, both mechanisms of positive feedback are expected to be at work. The relative importance of the two is expected to depend on the water infiltration properties of the soil. High infiltration will create a very thin layer of surface water, if at all, and drainage effects may be negligible. On the other hand, low infiltration will produce abundant surface water and drainage effects are expected to be dominant.

The five states, bare soil, spots, stripes, gaps and uniform vegetation, are asymptotic states, describing the behavior of the system at long times. The asymptotic spot and gap states consist of hexagonal arrays ( 0 and $\pi$ hexagons [11-13]). In practice the system may assume many more states. The coexistence of two or more asymptotic states allows for a wide variety of mixed states, where domains of one state are embedded in areas occupied by the other state. In addition, the system may be caught in long transients as environmental parameters vary on time scales similar to the relaxation times associated with the asymptotic states. Rainfall variations, for example, may increase the life time of rings as they evolve toward stripe patterns.

\section{Acknowledgments}

This study was supported in part by the Israel Science Foundation, grant $780 / 01$ 


\section{References}

[1] Cross MC and Hohenberg PC. Pattern formation outside of equilibrium. Rev. Mod. Phys., 1993; 65: 851-1112.

[2] Balmforth NJ and Provenzale A. Patterns of Dirt. Geomorphological Fluid Mechanics, Lecture Notes in Physics, edited by N.J. Balmforth and A. Provenzale. Berlin: Springer Verlag, 2001; 582: 369-393.

[3] Balmforth NJ, Provenzale A and Whitehead J. The language of pattern and form. Geomorphological Fluid Mechanics, Lecture Notes in Physics, edited by N.J. Balmforth and A. Provenzale. Berlin: Springer Verlag, 2001, 582: 3-33.

[4] Wettlaufer JS. Ice Surfaces: Macroscopic effects of microscopic structure. Phil. Trans. Roy. Soc. A, 1999; 357: 3403-25.

[5] Valentin C, d'Herbès JM, and Poesen J. Soil and water components of banded vegetation patterns. Catena, 1999;37:1-24.

[6] Catena Vol. 37: Special issue devoted entirly to banded vegetation.

[7] Klausmeier CA. Regular and irregular patterns in semiarid vegetation. Science, 1999; 284: 1826-1828.

[8] HilleRisLambers R, Rietkerk M, van den Bosch F, Prins HHT and de Kroon H. Vegetation pattern formation in semi-arid grazing systems. Ecology, 2001; 82: $50-? ? ?$.

[9] Thiéry J, d'Herbès JM and Valentin C. A model simulating the genesis of banded vegetation patterns in Niger. Journal of Ecology, 1995; 83: 497-507.

[10] Dunkerley DL. Banded vegetation: development under uniform rainfall from a simple cellular automaton model. Plant Ecology, 1997; 129: 103-111. 
[11] Lefever R and Lejeune O. On the Origin of Tiger Bush. Bull. Math. Bio., 1997; 59: 263-294.

[12] Lejeune $\mathrm{O}$ and Tlidi M. A model for the explanation of vegetation stripes (tiger bush). J. Veg. Sci., 1999; 10: 201-208.

[13] Lefever R, Lejeune O and Couteron P. Generic modelling of vegetation patterns. A case study of Tiger Bush in sub-Saharian Sahel. Mathematical Models for Biological Pattern Formation, IMA, edited by Maini PK and Othmer HG. New York: Springer, 2000; 121: 83-112.

[14] Rietkerk M, Boerlijst MC, van Langevelde F, HilleRisLambers R, van de Koppel J, Kumar L, Prins HHT and de Roos AM. Self-organization of vegetation in arid ecosystems. To appear in American Scientist.

[15] Murray JD. Mathematical Biology. Berlin: Springer Verlag, 1989, pp. 767.

[16] Wilson JB and Agnew ADQ. Positive feedback switches in plant communities. Advances in Ecological Research, 1992; 23: 263-336.

[17] Bare soils in arid regions are often covered by physical and biological crusts which reduce the infiltration of surface water into the soil. The physical crusts are consequences of the high impact of the rain at the ground level, and the biological crusts are often created by cyanobacteria. In vegetated areas the impact of the rain at the ground level is eliminated and the sun light is blocked. As a result the crusts do not develop and the infiltration remains high.

[18] Von Hardenberg J, Meron E, Shachak M and Zarmi Y. Diversity of vegetation patterns and desertification. Phys. Rev. Lett., 2001; 87: 198101-1 - 198101-4.

[19] Hillel D. Environmental Soil Physics. San Diego: Academic Press, 1998, pp. 771.

[20] Lange OL and Nobel PS. Physiological Plant Ecology II, edited by Osmond CB and Ziegler H. Berlin: Springer Verlag, 1982, pp. 747. 
[21] Coexistence of bare soil and uniform vegetation coverage have also been found in a one-variable model. See Ref. [11-13].

[22] Helld'n U. Desertification monitoring: Is the desert encroaching? Desertification Control Bulletin, 1988; 17: 8-12. 


\section{Figure Captions}

Figure 1: A bifurcation diagram for uniform states, showing the instability of the bare-soil state as the precipitation $p$ exceeds a critical value $p_{c}$. The vertical axis, $n$, represents here the biomass of uniform states. Solid lines denote states which are stable to uniform perturbations. Dashed lines denote unstable states. Beyond the instability, the bare-soil state evolves toward a uniform vegetation state. The instability is supercritical for $\rho<\rho_{c}$ and subcritical for $\rho>\rho_{c}$, where $\rho_{c}=3.8418$ for the parameters used in this study (see Sec. 2).

Figure 2: Growth rate curves, $\sigma=\sigma(k)$, for perturbations with wavenumbers $k$ around the uniform bare-soil state for three precipitation values, below the instability of the bare-soil state $\left(p<p_{c}\right)$, at the instability $\left(p=p_{c}\right)$ and above it $\left(p>p_{c}\right)$. The fastest growing mode at $p>p_{c}$ is characterized by a zero wavenumber and the growth is monotonic. The instability leads to a uniform vegetation state.

Figure 3: Growth rate curves, $\sigma=\sigma(k)$, for perturbations with wavenumbers $k$ around the uniform vegetation state for three precipitation values, below the instability of uniform vegetation $\left(p>p_{2}\right)$, at the instability $\left(p=p_{2}\right)$ and beyond it $\left(p<p_{2}\right)$. The fastest growing mode at $p<p_{2}$ is characterized by a finite wavenumber $k_{c}$ and the growth is monotonic. The instability leads to a stationary gap pattern.

Figure 4: Spatially averaged biomass $\langle n\rangle$ vs. precipitation $p$, obtained by simulating Eqs. (3) and (4). Linearly stable (unstable) solutions are represented by solid (dashed) lines. The line $\mathcal{B}$ represents the bare state $\langle n\rangle=0$. The curved line $\mathcal{V}$ designates the uniform vegetation state. The insets show typical patterns associated with the different nonuniform solution branches denoted by the colored lines. Parameter values are as specified in Sec. 2 . 
Figure 5: Patterns involving spots and stripes in a parameter range where both spot and stripe patterns are stable states of the system. (a) Coexistence of spot and stripe in spatially separated domains. (b) A mixed state of spots and stripes. The initial conditions in (a) where chosen so that the upper part converges to stripes and the lower part to spots. The interface between the two patterns appears to be stationary. In (b) the initial conditions consist of random perturbations about the unstable uniform vegetation state. Parameter values are as specified in Sec. 2 .

Figure 6: The development of spot patterns from two different initial conditions: (a) random perturbations of the unstable uniform vegetation state, and (b) three scattered spots. Isolated spots evolve first into rings which subsequently break into spots. Parameter values are as specified in Sec. 2.

Figure 7: The development of stripe or labyrinthine patterns from two different initial conditions: (a) random perturbations of the unstable uniform vegetation state, and (b) three scattered spots. The spots evolve first into rings before additional stripes appear. Parameter values are as specified in Sec. 2 .

Figure 8: Patterns of Paspalum vaginatum observed in the Northern Negev (200 mm mean annual rainfall): a labyrinth-like pattern (a) and closeups showing spots (b), stripes (c) and gaps (d). The typical distance between spots and stripes is about $0.1 \mathrm{~m}$.

Figure 9: Patterns of Poa bulbosa observed in the Northern Negev $(200 \mathrm{~mm}$ mean annual rainfall): scattered spots (a) and rings (b). Spot and ring diameters are in the range of $0.5-0.15 \mathrm{~m}$. 


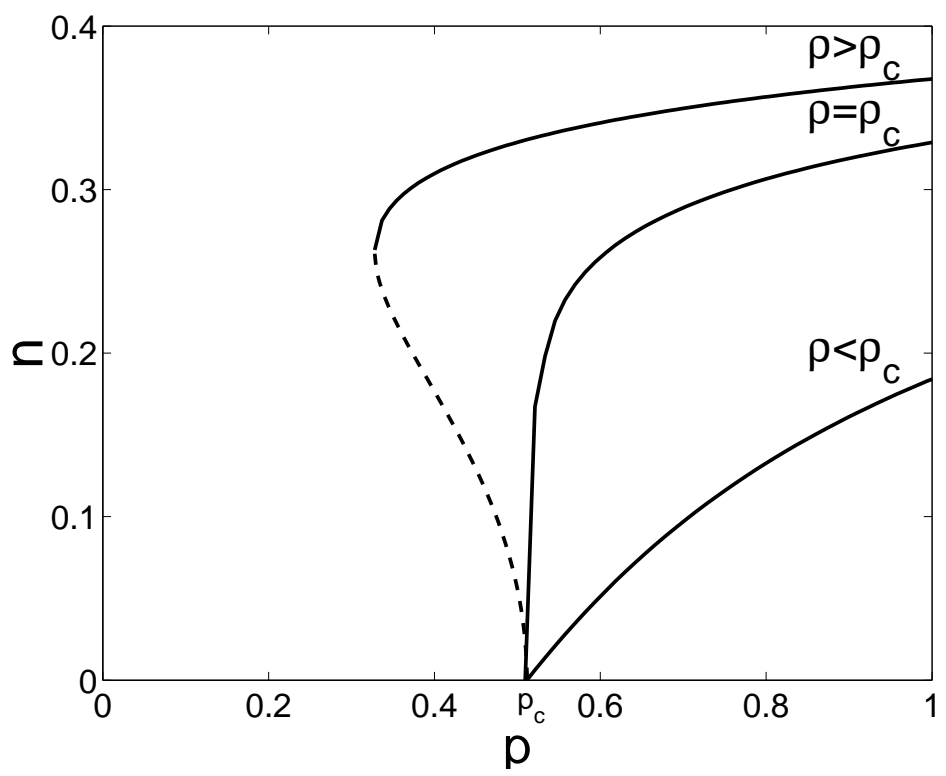

Fig. 1. A bifurcation diagram for uniform states, showing the instability of the bare-soil state as the precipitation $p$ exceeds a critical value $p_{c}$. The vertical axis, $n$, represents here the biomass of uniform states. Solid lines denote states which are stable to uniform perturbations. Dashed lines denote unstable states. Beyond the instability, the bare-soil state evolves toward a uniform vegetation state. The instability is supercritical for $\rho<\rho_{c}$ and subcritical for $\rho>\rho_{c}$, where $\rho_{c}=3.8418$ for the parameters used in this study (see Sec. 2). 


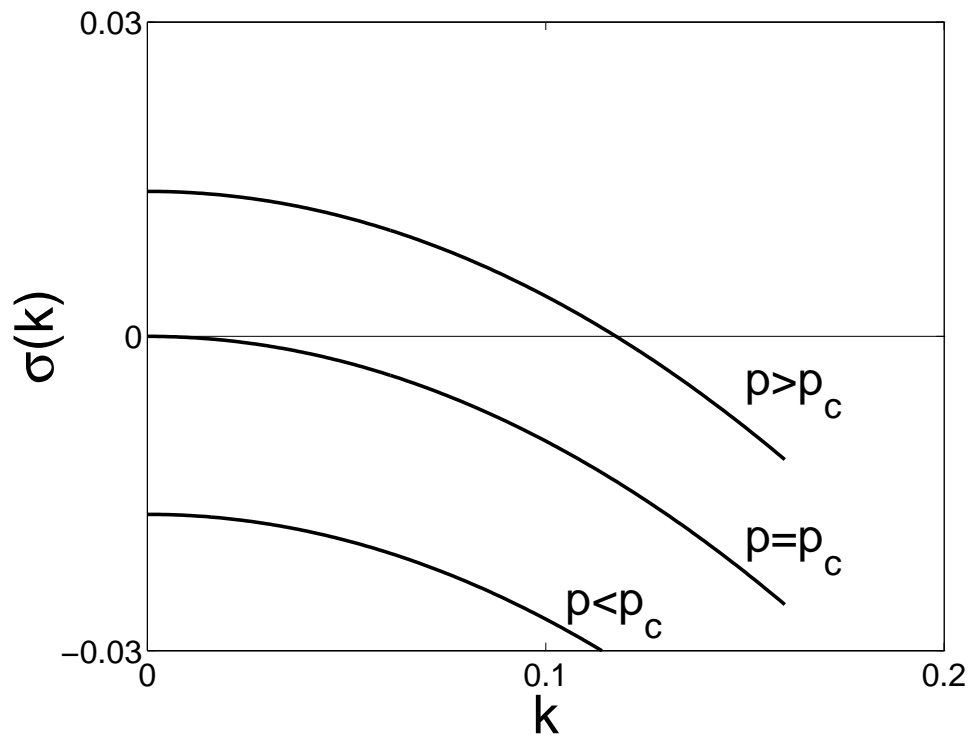

Fig. 2. Growth rate curves, $\sigma=\sigma(k)$, for perturbations with wavenumbers $k$ around the uniform bare-soil state for three precipitation values, below the instability of the bare-soil state $\left(p<p_{c}\right)$, at the instability $\left(p=p_{c}\right)$ and above it $\left(p>p_{c}\right)$. The fastest growing mode at $p>p_{c}$ is characterized by a zero wavenumber and the growth is monotonic. The instability leads to a uniform vegetation state. 


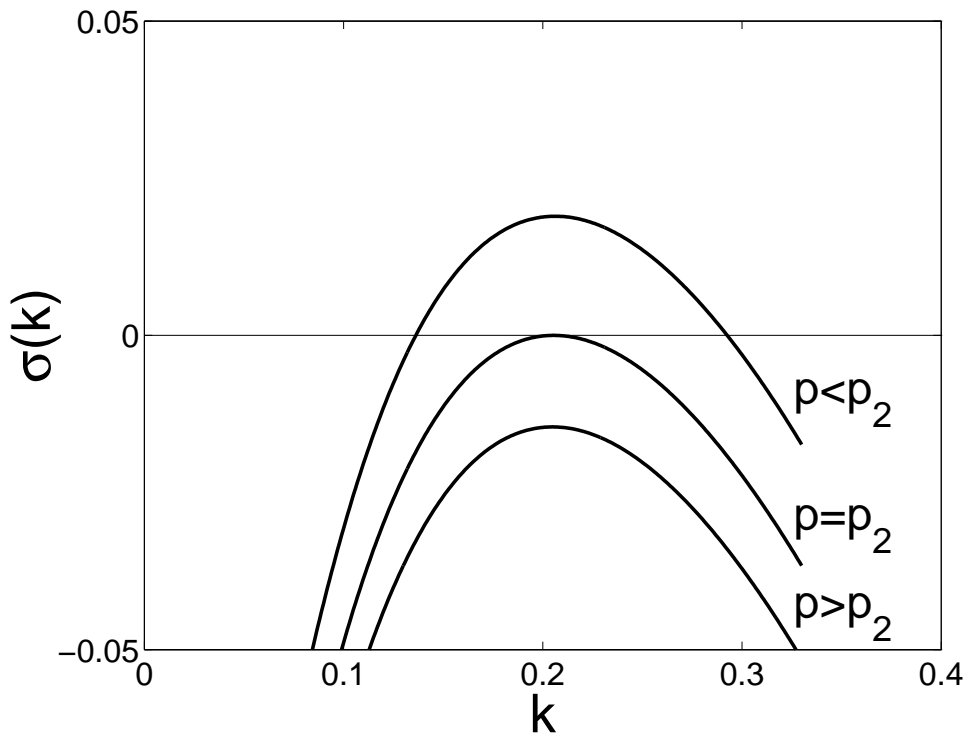

Fig. 3. Growth rate curves, $\sigma=\sigma(k)$, for perturbations with wavenumbers $k$ around the uniform vegetation state for three precipitation values, below the instability of uniform vegetation $\left(p>p_{2}\right)$, at the instability $\left(p=p_{2}\right)$ and beyond it $\left(p<p_{2}\right)$. The fastest growing mode at $p<p_{2}$ is characterized by a finite wavenumber $k_{c}$ and the growth is monotonic. The instability leads to a stationary gap pattern. 


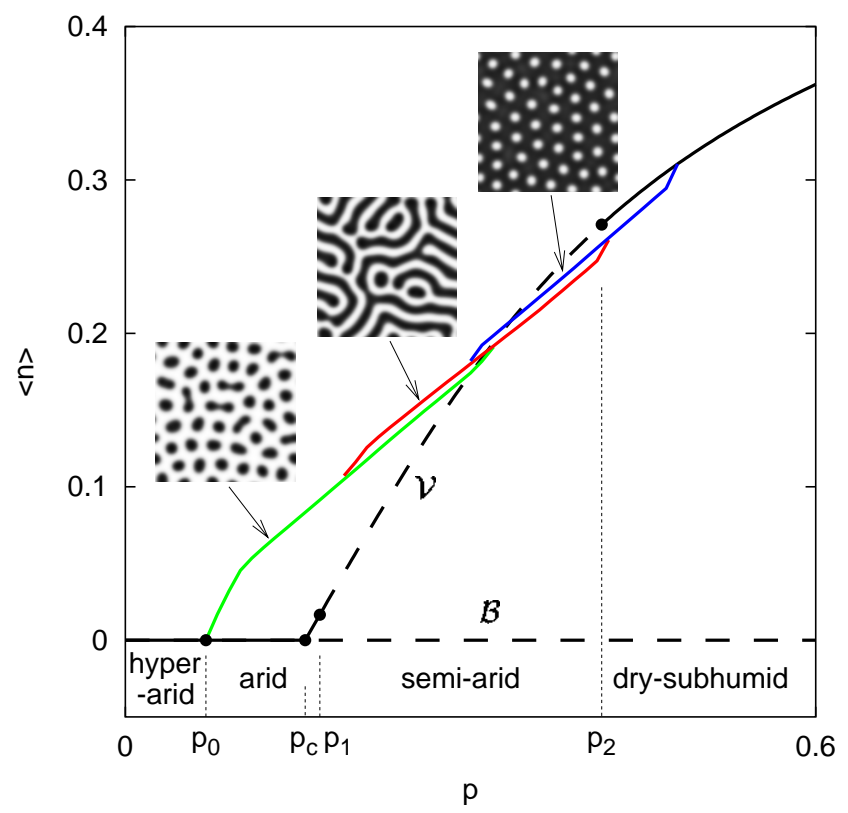

Fig. 4. Spatially averaged biomass $\langle n\rangle$ vs. precipitation $p$, obtained by simulating Eqs. (3) and (4). Linearly stable (unstable) solutions are represented by solid (dashed) lines. The line $\mathcal{B}$ represents the bare state $\langle n\rangle=0$. The curved line $\mathcal{V}$ designates the uniform vegetation state. The insets show typical patterns associated with the different nonuniform solution branches denoted by the colored lines. Parameter values are as specified in Sec. 2. 
a)

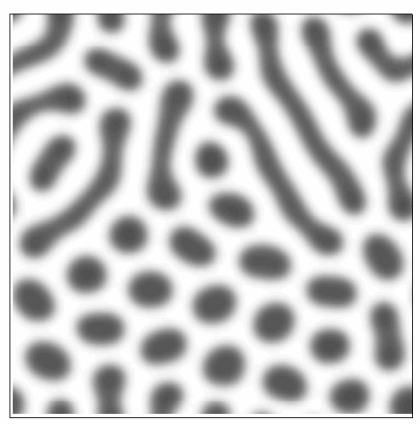

b)

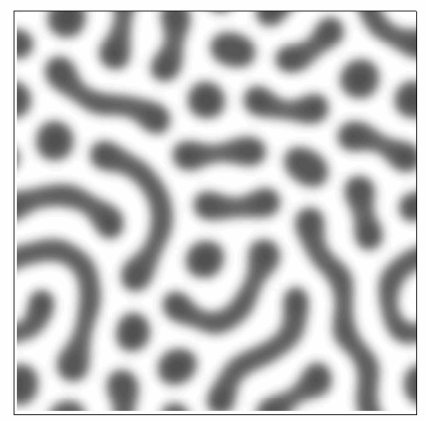

Fig. 5. Patterns involving spots and stripes in a parameter range where both spot and stripe patterns are stable states of the system. (a) Coexistence of spot and stripe in spatially separated domains. (b) A mixed state of spots and stripes. The initial conditions in (a) where chosen so that the upper part converges to stripes and the lower part to spots. The interface between the two patterns appears to be stationary. In (b) the initial conditions consist of random perturbations about the unstable uniform vegetation state. Parameter values are as specified in Sec. 2. 

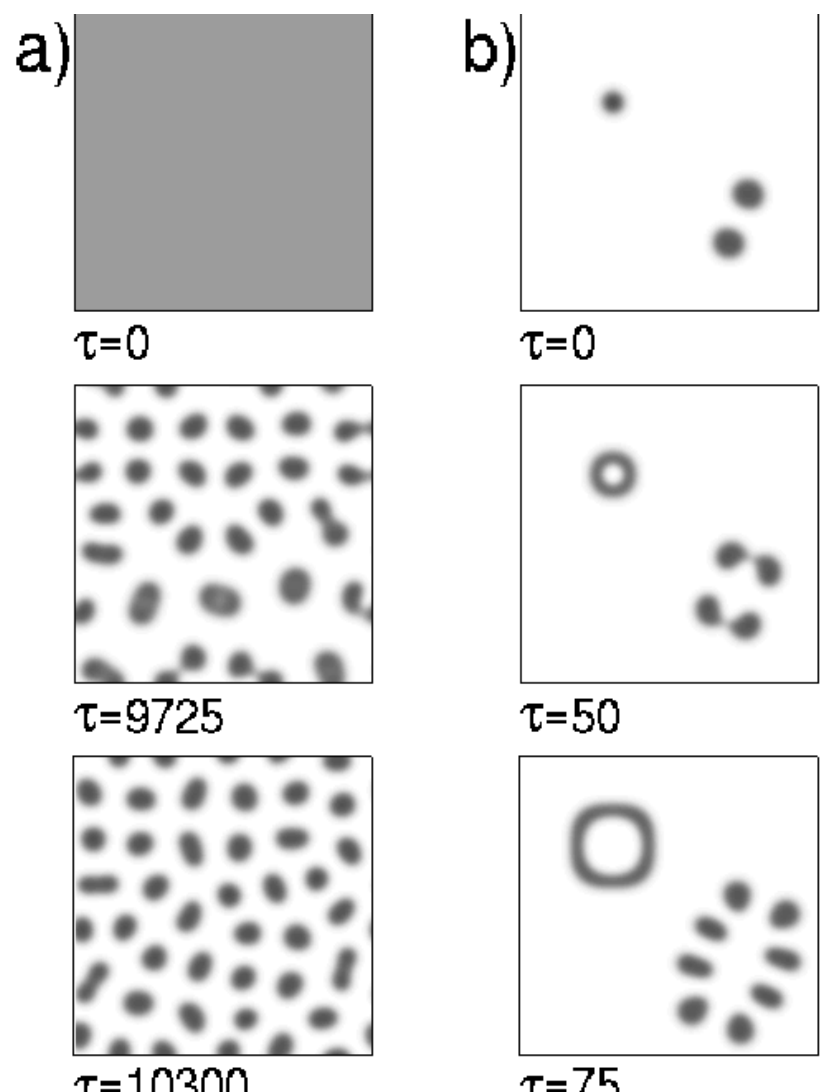

$\tau=75$
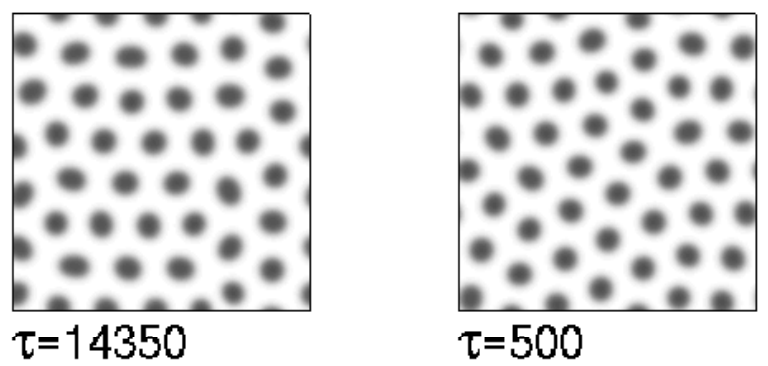

Fig. 6. The development of spot patterns from two different initial conditions: (a) random perturbations of the unstable uniform vegetation state, and (b) three scattered spots. Isolated spots evolve first into rings which subsequently break into spots. Parameter values are as specified in Sec. 2. 

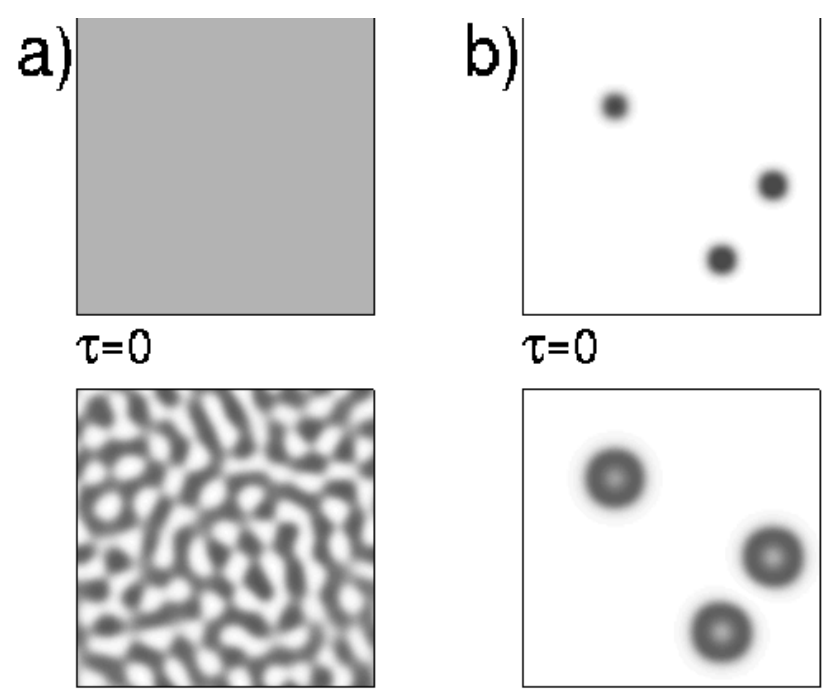

$\tau=150$

$\tau=37.5$
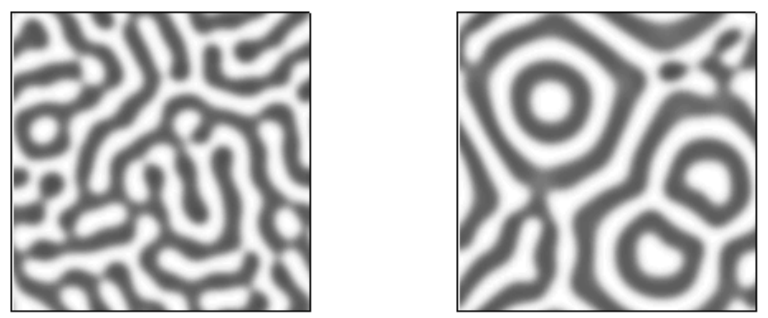

$\tau=225$
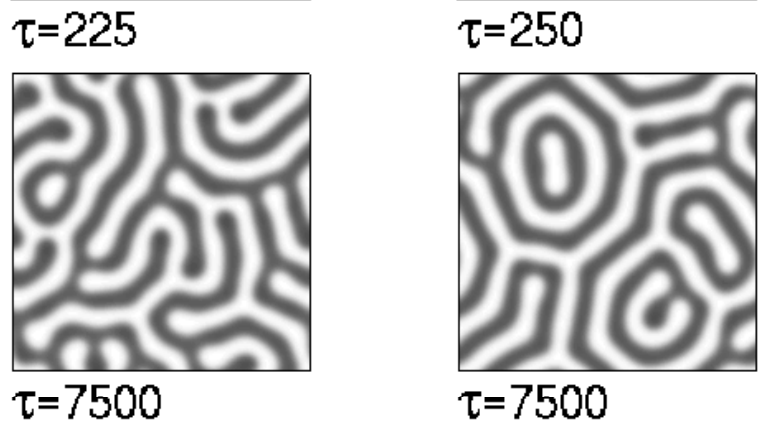

Fig. 7. The development of stripe or labyrinthine patterns from two different initial conditions: (a) random perturbations of the unstable uniform vegetation state, and (b) three scattered spots. The spots evolve first into rings before additional stripes appear. Parameter values are as specified in Sec. 2. 


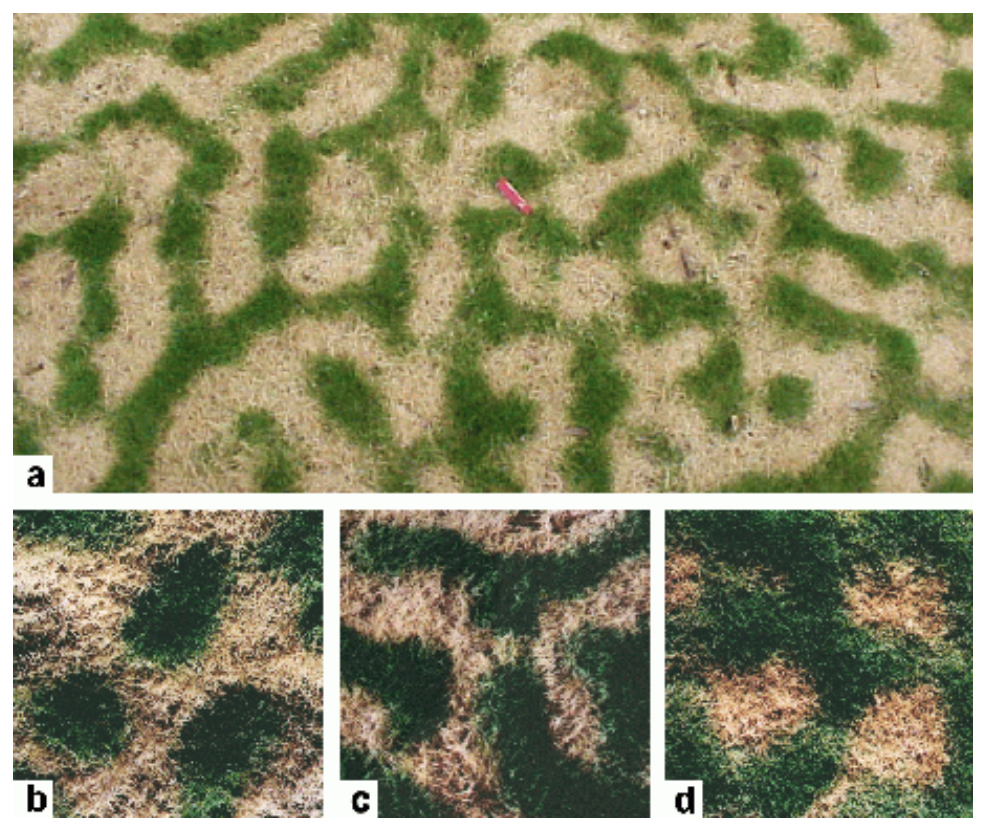

Fig. 8. Patterns of Paspalum vaginatum observed in the Northern Negev $(200 \mathrm{~mm}$ mean annual rainfall): a labyrinth-like pattern (a) and closeups showing spots (b), stripes (c) and gaps (d). The typical distance between spots and stripes is about $0.1 m$. 


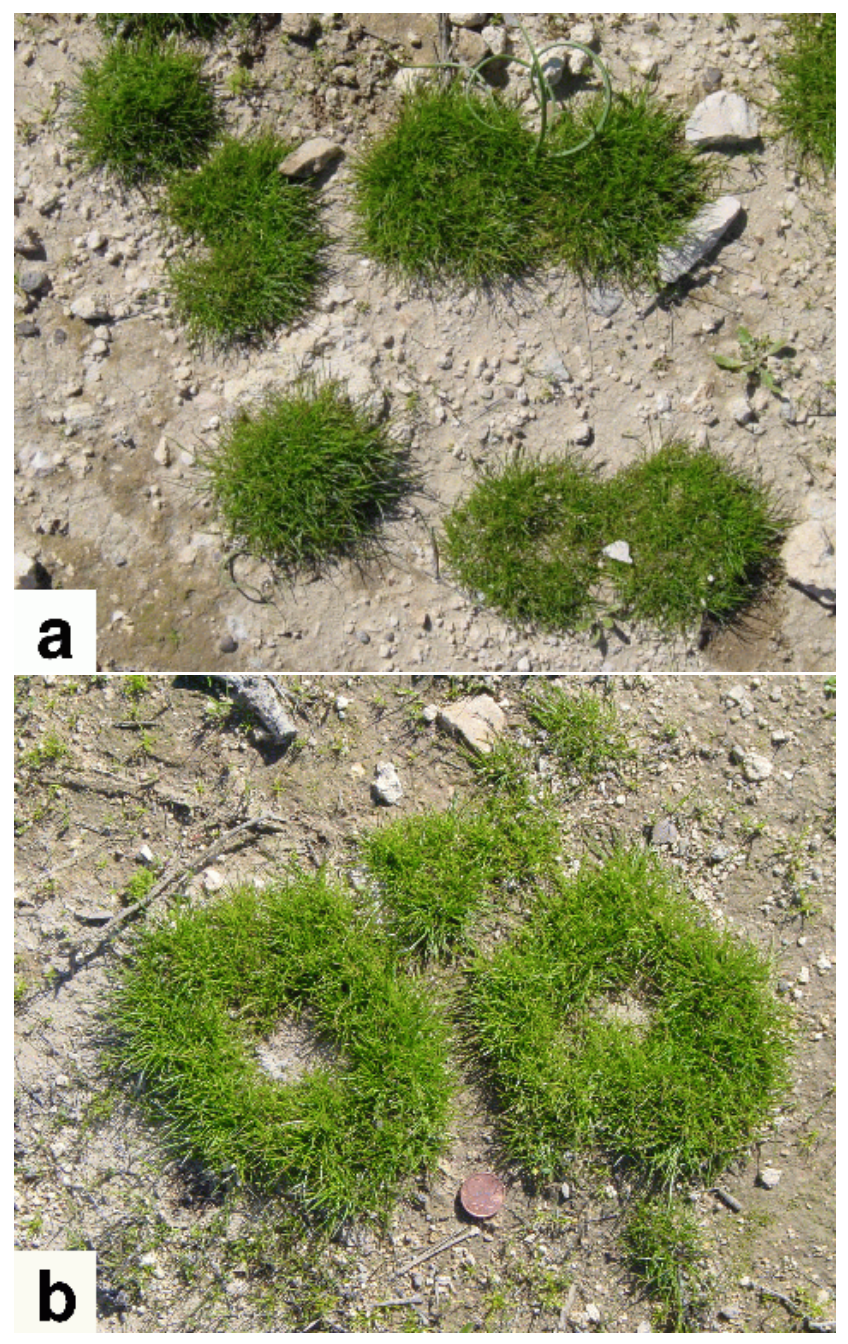

Fig. 9. Patterns of Poa bulbosa observed in the Northern Negev (200 $\mathrm{mm}$ mean annual rainfall): scattered spots (a) and rings (b). Spot and ring diameters are in the range of $0.5-0.15 \mathrm{~m}$. 